\title{
Methods for the Sustainable Design of Solar Energy Systems for Industrial Process Heat
}

\author{
Alessandro Franco \\ Department of Energy, Systems, Territory and Constructions Engineering, University of Pisa, \\ Largo Lucio Lazzarino, 56126 Pisa, Italy; alessandro.franco@ing.unipi.it
}

Received: 19 May 2020; Accepted: 17 June 2020; Published: 23 June 2020

\begin{abstract}
In this paper, the production of low to medium temperature water for industrial process heat using solar energy is considered. In particular, the paper outlines the perspective of an optimum design method that takes into account all of the typical variables of the problem (solar irradiation, system architecture, design constraints, load type and distribution, and design and optimization criteria) and also considers the use of the fossil fuel backup system. The key element of the methodology is the definition of a synthetic combined energetic and economic utility function. This considers the attribution of an economic penalty to irreversibility in connection with the use of a fossil fuel backup. This function incorporates the share of the solar system production (solar fraction) as an optimum design variable. This paper shows how, using the proposed criteria, the optimal value of the solar fraction, defined as the share of operation of the solar system with respect to the whole energy demand, can be increased. Current practice considers values in the range between 40 and $60 \%$. However, levels up to $80 \%$ can also be obtained with the proposed methodology. Thus, penalizing the use of fossil fuels does not exclude a priori their contribution.
\end{abstract}

Keywords: solar energy; industrial process heat; flat plate solar collectors; Second Law Analysis; solar fraction; optimum design

\section{Introduction}

One of the crucial elements for increasing the sustainability of an energy system is the reduction of carbon dioxide emissions. A relevant contribution is the increase of renewable energy use with a consequent reduction of fossil fuel consumption. Renewable Energy Systems (RES) can be used for supplying both process heat and electricity in the civil and industrial sectors. Since heat currently accounts about $50 \%$ of final energy demand in the European Union, a significant contribution from the renewable heating sector is still expected. Solar thermal systems are particularly interesting in terms of promoting a substantial increase of the share of low temperature heat produced by solar energy. Increasing the amount of process heat production for industrial applications using solar energy sources is of real importance. In some European countries, it is estimated that more than $25 \%$ of the total final energy demand is thermal energy in the form of hot water or steam for industrial processes. This is typically in the range between 60 and $250{ }^{\circ} \mathrm{C}$ [1]. An important aspect of such energy demands concerns water at medium to low temperature (between 60 and $100^{\circ} \mathrm{C}$ ). The use concerns various sectors like the food (meat, diary, beverages, and tinned food), paper, textile, and mechanical (chemical, automotive) industries, as well as pharmaceuticals and, in several cases, it corresponds to the production of hot water for cleaning purposes.

Various papers [2-4] have reviewed the topic. The authors analyze existing industrial processes in order to identify typical applications for solar process heat, and investigate future opportunities to integrate solar heat into industrial companies. The three above-mentioned papers, by different authors and published in the period from 2017 to 2019, provide a comprehensive review of the industrial 
processes that can adopt solar process heating systems, and thus help move towards sustainable production in certain industries. The solar systems are analyzed with respect to collector technologies and demand temperatures; all of the papers discuss the problem that, while solar thermal is already a very well-known and popular technology, only a few industries employ solar energy in industrial processes to generate process heat.

The problem is that, in general, loads and utilization profile are approximately constant throughout the year, while solar input is largely variable in particular seasons and at certain hours of the day. Moreover, the amount of energy required is relevant such that the initial investment and operating costs often discourage the investment.

Thus, notwithstanding the important increase in the use of renewable energy, to date, most energy has been produced using fossil fuel resources such as oil and natural gas. From an energy perspective, mainly taking into account the Second Law of Thermodynamics, this practice is highly questionable due to a series of motivations, in particular the high irreversibility associated with the operation of the boilers. High-quality energy (derived from fossil fuels as natural gas with high lower heating value) is used for obtaining an increase in water temperature by a small number of degrees (from the environmental temperature up to the temperature of final utilization, typically between 40 to $80^{\circ} \mathrm{C}$ ). From a theoretical point of view, this determines relevant energy degradation (irreversibility); this is caused by the entropy generation associated with heat transfer between the high temperature combustion gases (more than $1000^{\circ} \mathrm{C}$ ) and water at a temperature below $100^{\circ} \mathrm{C}$, and environmental impact.

In an alternative method, the energy required can be generated by solar thermal energy systems, contributing both to a further increase in the use of RES and to the reduction of entropy generation, driving towards sustainable production in industries $[5,6]$. If a solar system for domestic applications has undergone relevant development in recent years, the same cannot be said for systems for producing "solar" heat for industrial processes. This is a relevant field of analysis from the perspective of future development of solar energy; it is not always simple to promote such energy, both for technical and economic reasons [7].

Interesting opportunities for the integration of solar thermal systems in industrial processes can be identified in a series of fields, for which the use of water or air at a temperature of $50-80{ }^{\circ} \mathrm{C}$ is required. Examples include the heating of water for washing or cleaning purposes.

For a defined profile of the thermal load, the design of a solar thermal system consists of the definition of the area of the solar collectors (in general, flat plate collectors), the size of the storage volume, and the fossil fuel based thermal integration system (auxiliary boilers).

Because the solar thermal energy profile is periodical, with important variation during the year, and the load profile of the various industries is approximately constant, solar thermal energy systems can be integrated into the processes directly without a network of additional heat exchangers. A fossil fuel-based backup system is necessary, and this constitutes a significant source of $\mathrm{CO}_{2}$ emissions. In general, the result is a solar production ratio ranging from 40 to $60 \%$ of the annual total energy amount. As mentioned previously, a solar thermal energy system of this type requires an additional thermal input from one or more auxiliary devices (such as an auxiliary boiler) that operate mainly during winter and mid-seasons. From the point of view of the Second Law of Thermodynamics, the operation of the auxiliary boiler is highly inefficient due to its high irreversibility (entropy production).

The design methods for a Solar Water Heating System (SWHS) are analyzed diffusely in the literature. In the past, various papers, such as [8-14], have proposed a design method for SWHSs based on statistical considerations. Since the late 1990s, it has been possible to find methods for obtaining the optimum design. A paper by Kulkami et al. [15] represents a typical example of such an approach.

In recent years, the analysis of solar energy systems, often for the civil and residential sector, has been characterized by the use of commercial software, such as TRNSYS, [16,17], or self-developed code based on commercial platforms, such as Aspen-HYSIS [18]. A Python program has been developed and used to calculate the levelized costs of differently designed solar heating systems [19]. 
The analysis of the literature clearly highlights evidence of the problems connected with an efficient use of solar systems. This is often related to a kind of economic optimization of the system, in which the objective function is economic (minimization of the levelized cost of energy), technical (maximization of an overall performance index), or environmental (minimization of pollutant emissions), applied individually or collectively, as discussed in [20].

It is evident that the development of methods and indicators that try to combine economic and energetic or environmental (maximization of the solar energy fraction) objectives, could be useful for increasing the use of solar generation for industrial process heat and, for a single plant, the share of utilization of solar energy with respect to the total energy required.

After a preliminary analysis of the literature on the design of a solar thermal heating system, mainly for industrial process heat generation, this paper focuses on possible alternative design strategies, with the objective of increasing the solar production rate associated with the systems. The optimum design strategy proposed pursues a multi-objective optimization obtained by combining a reduction in the total irreversibility, via an increase of the solar operation share and connected to a reduction of fossil fuel use for backup (perspectives of the Second Law of Thermodynamics), and providing more general economic convenience.

The original element of discussion proposed in this paper is the definition of a specific objective function, including a penalization term for irreversibility, and the consideration of the share of operation of the solar system as the independent variable. Irreversibility arises due to the operation of the integrative (fossil fuel based) systems and, consequently, the additional term introduced represents a penalty to the use of fossil fuel. The auxiliary boiler often has a high First Law Efficiency (higher than 95\%), but low values of Second Law Efficiency (often lower than 0.1), connected to relevant energy degradation (from "high quality" energy of the input fuel to low quality energy represented by water at temperatures below $100^{\circ} \mathrm{C}$ ).

The original contributions of this paper are the proposal of a relatively simple approach, and the idea of reducing the environmental impact related to fossil fuel use (both in terms of energy source consumption and consequent carbon dioxide emissions) to a simple synthetic indicator. Compared to a conventional economic analysis, the method has the objective of determining an increase of the size and operating time of the solar thermal system with respect to the operation of the auxiliary boilers, contributing to an increase of the solar fraction of the thermal system, thereby offering a possible convenience. In the final part of this paper, the proposed methodology is tested by referring to a specific case. A further element discussed in the work is the idea of a possible modification of the economic support systems for the diffusion of the production of process heat based on a solar energy system.

\section{The Potential for Solar Thermal Energy Use in Industrial Sector: General Elements}

As discussed in the previous section, solar heat for domestic applications has increased in market share in various countries, while solar process heat, mainly for various industrial applications, appears to be less developed. One of the problems is that, even in countries where subsidy programs covering solar thermal process heat installations exist, the return of the investment is often long relative to the wider electricity sector, for example, with photovoltaic (PV) plants. Moreover, an additional problem is that in many regions the subsidy programs connected to thermal energy are frequently changing because a clear strategy has not been adequately identified.

In principle, the potential is clear: A considerable portion of total industrial heat demand occurs at a temperature below $100{ }^{\circ} \mathrm{C}$, and it is possible that a large amount of this demand could be met with commercially available flat plate solar thermal collectors (FPCs). Solar process heat can be technically and economically viable in a variety of industrial processes, such as cleaning and washing, heating, drying, and pre-heating. In a recent paper, Kalki et al. analyze the various methods used by engineers in the design of SWHSs for process heat production in manufacturing systems and the related problems [21]. A crucial element is the temperature required; it is relatively simple to define the size of a solar thermal system when the process temperature is below $60^{\circ} \mathrm{C}$, while a temperature 
above $60^{\circ} \mathrm{C}$ requires large collector surfaces and storage systems, or a consistent energy supply from fossil fuels. In this case, a scheme including an auxiliary boiler is required.

Figures 1 and 2, adapted from textbook [6], represent the two most relevant reference configurations, and include the three main components of the systems: Solar collectors, storage volumes, and auxiliary boilers. As reported in [6], more complex configurations are available, including those with additional heat exchangers, but these are not relevant for the purpose of the present paper.

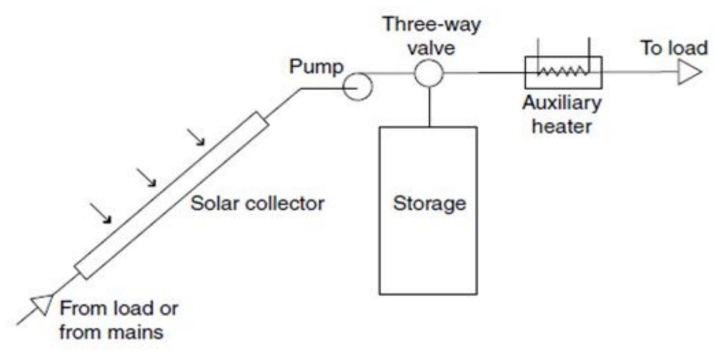

Figure 1. Solar Water Heating System (SHWS) configuration: Open loop.

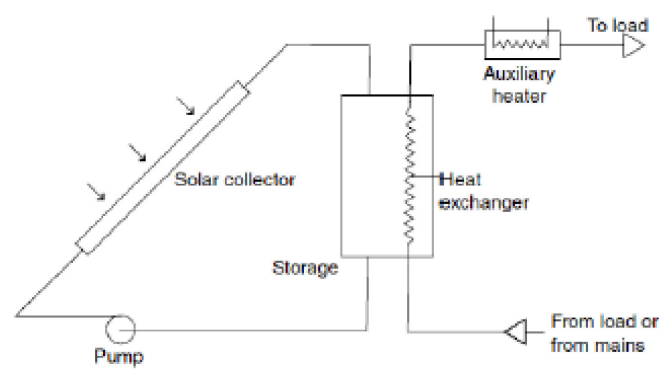

Figure 2. SWHS configuration: Closed loop.

Open loop systems, represented in Figure 1, appear to be a suitable solution for non-freezing, warm climates (where the risk of freezing is low). Simplicity and economy are the specific advantages of this system, in addition to higher efficiency, because the additional loss due to the heat exchanger is not present. Closed loop systems, as represented in Figure 2, are suitable for continental climates (from southern to central Europe). In this case, an anti-freeze solution is added to the carrier fluid, which is different from that going to the user. In both schematizations, the solar collector is the main component of the system and represents the most relevant cost. Low temperature collectors supply the carrier fluid at a temperature usually lower than $80^{\circ} \mathrm{C}$. Different from concentrating collectors, conventional FPCs work with both direct and diffused radiation.

The first prerequisite for success in a solar system application is an adequate insolation level. Figure 3 provides a typical trend of the average values of daily solar insulation on a horizontal surface; the values reported in Figure 3 are only indicative values, obtained using a typical mathematical model from [5]. 


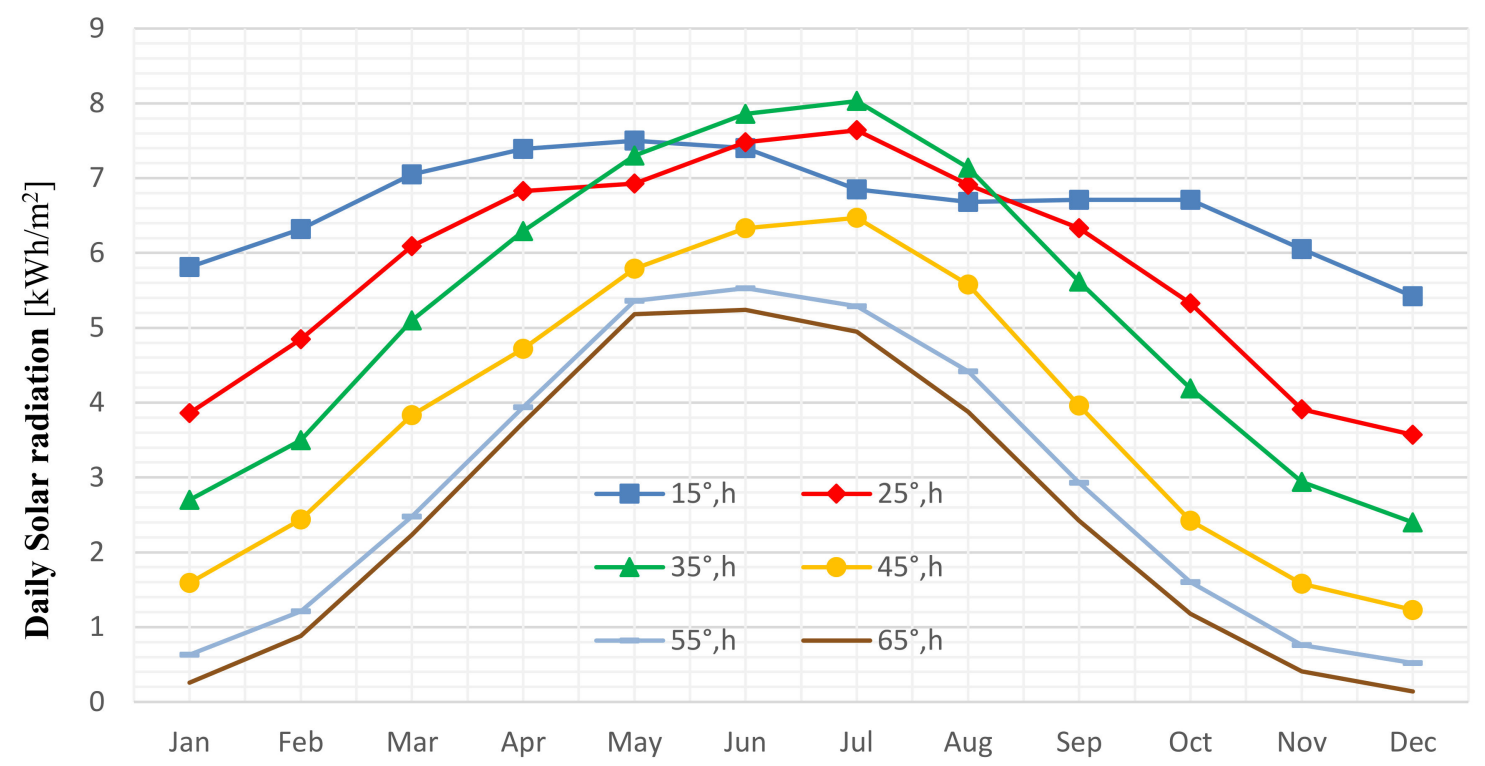

Figure 3. Average daily solar radiation per month on a horizontal (h) at different latitudes.

The use of solar energy is generally possible when a minimum irradiation of $1500-1600 \mathrm{kWh} / \mathrm{m}^{2}$ per year (corresponding to a mean daily insolation of $3.7-4 \mathrm{kWh} / \mathrm{m}^{2}$ per day) is available (for example, in Southern Europe or similar climatic conditions). If the irradiation level is reduced to $1000-1200 \mathrm{kWh} / \mathrm{m}^{2}$ per year, which can be observed in connection with a lower average environmental temperature, the use of solar energy, and solar FPCs in particular, is often less convenient.

While solar thermal energy-based systems such as SWHS are employed in industry for typical summer applications, the highest potential for the integration of solar thermal exists for industrial processes with the following characteristics:

- Demand for thermal energy for more than $80 \%$ of the year, including summer;

- demand for at least five days per week;

- daily demand in the summer period is sometimes lower than that of the rest of the year.

Considering these aforementioned elements, in order to define the specific requirements for thermal energy, the design of the solar thermal plant is the result of an economic analysis.

The trade-off size is obtained as a balance between the cost of the system and the cost of the fossil fuel input. For this reason, and due to the fact that the surface of the collectors is often large, the convenience of the solar thermal plant is clear only if economic support is available and, in general, the share of solar operation has often been below $50 \%$. For the design of a SWHS for industrial process heat, the next steps are recommended:

- define an overall thermal load profile (temporal distribution of the thermal load);

- identify one or more possible configurations of the solar thermal system;

- $\quad$ determine the collector area $(\mathrm{CA})$, the storage volume $(\mathrm{V})$, and the size of backup system $\left(\mathrm{P}_{\mathrm{th}}\right)$;

- perform operative simulations modifying the area of the collector field, the type of collector, and the storage volume, thereby obtaining different values for the "solar fraction" of the system.

As suggested in the practical design guidelines for solar energy systems, such as [22,23], the performance of the thermal solar system depends on the following variables or parameters:

- $\quad$ Running conditions (final temperature required, $T_{\text {out }}$; and temperature increase, $\mathrm{T}$ );

- climatic conditions (environmental temperature, solar irradiation profile);

- $\quad$ energy input for the operation of the fossil fuel back-up systems (oil, gas, electricity, or other fuel); 
- the thermal load and heat demand (mass flow rate or mass of water required in the unit time, and temporal profile);

- solar collector technical characteristics (type, area, efficiency curve, cost, etc.), according to the market standards;

- $\quad$ storage system characteristics (type of storage volume, size, etc.);

- collector loop losses and transmission losses between storage and backup systems (which strictly depend on the total length, type of insulation, heat exchanger efficiency, etc.);

The relevance of the different elements depends on the type of system, the location, the running conditions (temperature, control, sizing), and the control strategy. Due to the possible variation of demand and solar radiation, and considering that energy production is required in each month of the year, the solar thermal system is equipped with an auxiliary boiler operating with a fossil fuel (in general, oil or natural gas) or sometimes electricity, in order to provide heat integration. An important element is the solar share or solar fraction of the system, $s f$, defined as the ratio between thermal energy produced with solar energy input and the total energy required for the specific application under analysis:

$$
s f=E_{Q, \text { solar }} / E_{Q, \text { total }}
$$

The solar fraction depends on different factors, such as collector type and size, heating load, storage volume, and available solar radiation. In a large number of cases observed, the contribution of solar energy (i.e., the value of the solar fraction) is a reduced amount of the total energy required (sometimes less than 30\%). This means that the operation of the auxiliary boiler is high and, consequently, exergy losses (energy degradation) are associated with the operation of the system. On the other hand, solar thermal systems with an annual solar fraction of $100 \%$ may not be convenient, for different reasons. A break point is often obtained when using an economic criterion, where it is observed that an additional increase in collector area does not result in a significant increase in the obtained heat load; this may be due, for example, to the relevant amount of energy wasted during the summer period. Methods to obtain a trade-off point are proposed, for example, in [24,25].

\section{The Design of Solar Water Heating Systems (SWHS): Criteria and Guidelines}

Designing a SWHS involves appropriate sizing of the different components, which are based on predicted solar isolation and hot water demand. There are various methods for the design of SWHS. An overview of these is available in textbooks, such as [26], and technical reports, such as [22,23]. Criteria with more severe conditions would lead to an intolerable restriction of the field which, due to the cost of the collectors, would cause bankruptcies without ensuring full autonomy. Correct sizing is therefore not only a technical problem; a combined technical and economic analysis often permits an optimized solution to be obtained.

The steps of solar system design are the following:

- Calculation of available radiation;

- $\quad$ estimation of the thermal load;

- $\quad$ sizing of the solar field;

- $\quad$ sizing of the tank;

- calculation of the solar fraction on a monthly and annual basis;

- $\quad$ sizing of the components of the solar circuit (heat exchanger, expansion tank, etc.).

The design of a SWHS requires preliminary knowledge of the thermal load and its distribution. This could be continuous or variable throughout a single day or during various seasons. The starting point of a good design is an exact knowledge of the thermal load of the user.

Different methods have been developed in the literature for designing solar energy systems. These range from those based on detailed simulation, such as the day-by-day iterative method (an iterative 
process extended over 365 days/year), to more simple design methods, such as the f-chart method, where $\mathrm{f}$ is the fraction of the heating load supplied by solar energy during each month. Other rule of thumb methods also exist [4].

For defining the size of the various components of the SWHS, it is important to know the mean daily demand of hot water, since the daily or weekly loads can be different. The amount of energy obtained by solar energy with respect to that obtained by fossil fuel (solar fraction) depends on available insolation and the efficiency of the solar collectors. Insolation differs widely and is crucial for each solar system.

SWHS efficiencies depend on the collector types, the solar radiation intensity, and the temperature required for the water. Thermal losses are clearly minimal if the temperature of the water used for the application is close to the ambient temperature and the intensity of radiation is close to the maximum value (about $1000 \mathrm{~W} / \mathrm{m}^{2}$ ), while efficiency is reduced when temperature increase and intensity of radiation decrease.

The conventional flat plate collector is the core element of a solar system. Low temperature panels supply the carrier fluid at a temperature usually less than $80^{\circ} \mathrm{C}$. The use of a simple flat plate absorber without glazing can achieve the highest efficiencies, of up to $90 \%$, while more structured collectors with evacuated tubes or multiple covering elements reduce the maximum efficiency level. In general, the combination of collector efficiency and solar irradiation yields the production rate of the solar collector. Typical characteristics of the most common types of solar collectors, according to the European normative, can be grouped as shown in Table 1.

In cases such as the one described above, the sizing of the solar collectors (area) and the storage system (volume) is based on the assumption of a fixed share of the annual thermal load produced by solar energy: The assumed minimum and maximum values are $40 \%$ and $60 \%$, respectively. In this way, the operation of the auxiliary boiler, using fossil fuel, is important, so that solar energy permits the total amount of the thermal load to be obtained only during summer, and a reduced amount $(10-15 \%)$ during the cold season.

Table 1. Typical values for solar radiation and collector production. FPC $=$ flat plate solar thermal collectors.

\begin{tabular}{ccccc}
\hline \multirow{2}{*}{ Purpose } & \multirow{2}{*}{ FPC Type } & \multirow{2}{*}{$\mathbf{T}_{\mathbf{W}}{ }^{\circ} \mathbf{C}$} & \multicolumn{2}{c}{ Production $\mathbf{k W h} / \mathbf{m}^{2} /$ Year } \\
\cline { 3 - 5 } & & & North Europe & South Europe \\
\hline Low T & unglazed & $30-70$ & $250-450$ & $450-650$ \\
Medium T & glazed & $50-100$ & $350-500$ & $500-800$ \\
\hline
\end{tabular}

Considering a case, such as the one illustrated in Figure 3 for example, for each $\mathrm{m}^{3}$ of water required for the industrial process, considering about 240 working days during the year, the estimated surface of the collectors can be expressed in $\mathrm{kWh}$ as:

$$
E_{Q, w d}=M_{W, w d} \cdot c_{p} \cdot \Delta T / 3600
$$

For a reference quantity of water required (e.g., $1 \mathrm{~m}^{3}=1000 \mathrm{~kg}$ ) and a typical value of $50^{\circ} \mathrm{C}$ for the temperature increase $T$, assuming $c_{p}=4.2 \mathrm{~kJ} / \mathrm{kg} \mathrm{K}$ for the specific heat of water, an amount of energy of about $52.5 \mathrm{kWh}$ (for each $\mathrm{m}^{3}$ of water required for the process) has to be produced.

For one year of operation, a total amount of $12,500-20,000 \mathrm{kWh}$, depending on the total days of operation (from a minimum of 200 to a maximum of 365), is required.

The corresponding surfaces of the collectors of the flat plate type, $A$, can be obtained considering the annual value of the energy required and the solar fraction fixed as a design objective, $s f$, depending on the net annual solar radiation, obtained with the collectors, $e_{s, n e t}$, as:

$$
A=E_{Q, \text { year }} \cdot s f / e_{s, \text { net }}
$$


The amount of heat collected with a solar collector that allows evaluation of the term $e_{s, n e t}$ in Equation (3) depends on the solar radiation, $G$, and on the collector efficiency, which for a FPC can be expressed as:

$$
\eta_{C}=\eta_{0}-\frac{a_{1}\left(T_{c}-T_{0}\right)+a_{2}\left(T_{c}-T_{0}\right)^{2}}{G}
$$

where $T c$ is an average collector air temperature, $T_{0}$ is the ambient temperature, and $G$ is the solar radiation incident on the collector surface, expressed as a specific power. For a first-order analysis, the temperature of the collector can be assumed to be a linear average value between the inlet temperature $\left(T_{\text {in }}\right)$ and the outlet temperature $\left(T_{\text {out }}\right)$ :

$$
T_{C}=\frac{T_{\text {in }}+T_{\text {out }}}{2}
$$

In particular, the value of $e_{s, n e t}$, expressed in $\mathrm{kWh} / \mathrm{m}^{2}$, is the result of the product of:

$$
e_{s, \text { net }}=\eta_{c, a v g} \cdot e_{s}=\eta_{c, a v g} \cdot \int_{0}^{8760} G \cdot d t
$$

In places where solar energy can be profitable, the values of $e_{s, n e t}$ range between 1200 and $1800 \mathrm{kWh} / \mathrm{m}^{2}$, and average efficiency values for the annual operation of a flat plate collector are 0.3-0.4. Thus, the value of the production rate, $e_{s, n e t}$, ranges from $360 \mathrm{kWh} / \mathrm{m}^{2}$ per year in typical northern European conditions to $720 \mathrm{kWh} / \mathrm{m}^{2}$ per year in typical southern European conditions. This results in an average production rate of $500 \mathrm{kWh} / \mathrm{m}^{2}$ for each year (lower limit value for Southern European conditions, typical, for example, of northern Italy) and a solar fraction ( $s f$ ) ranging between 0.4 and 0.6 .

Considering the above data, it is possible to obtain a reference area value for each cubic meter of water required in a single working day of:

$$
\begin{array}{ll}
\left.\mathrm{a}=7 \div 14 \mathrm{~m}^{2} \text { (reference value } 10 \mathrm{~m}^{2}\right) & \text { if } \mathrm{sf}=0.4 \\
\left.\mathrm{a}=10.5 \div 21 \mathrm{~m}^{2} \text { (reference value } 15 \mathrm{~m}^{2}\right) & \text { if } \mathrm{sf}=0.6
\end{array}
$$

Moreover, it is important to contextually define the size of a storage volume that could be relevant in order to mitigate the effects of the time span between the production peak and the thermal power demand peak, and to reduce the amount of energy wasted.

A preliminary sizing of the storage volume can be obtained with simple direct calculation or using reference data, available from textbooks, such as [6], and technical manuals, such as [22,23].

For example, in typical central European conditions, a 50-L storage for each $\mathrm{m}^{2}$ of solar collector surface can be used, while in southern Europe, where the level of solar irradiation is higher, a value of $80 \mathrm{~L}$ of storage for each square meter of collector surface can be assumed.

The main drawback to the spread of solar thermal energy systems is the fact that active solar systems have to compete with conventional fossil fuel-based systems, for which the environmental costs are not fully considered. Therefore, in several cases, systems based on conventional fossil fuel energy inputs appear to be cheaper relative to active solar heating, and the widespread use of solar energy systems is only possible if a form of financial support is available. In general, solar thermal technology for production of industrial process heat does not emerge as a clear option because the economics of the solar heating systems cannot be considered to be particularly attractive. The cost of the various components differs by technology and varies considerably in different countries [27].

\section{Sizing of SWHS for Process Heat: Common Practice and the Method Proposed}

In general, for producing thermal energy in the form of hot water at temperatures of $40-80{ }^{\circ} \mathrm{C}$, boilers using fossil fuels such as natural gas are commonly used. It is generally difficult to replace these systems with renewable energy-based systems, such as those based on solar energy. 
Modeling techniques to evaluate the performance of solar systems requires, in principle, models for daily analysis of solar radiation and models for the operation of solar collectors and storage systems. Various methods of analysis are available in the previously mentioned papers and textbooks.

On the other hand, methods of analysis and optimization of energy systems have to take into account energy use and financial resources needed, in addition to the scarcity of physical resources used and any pollution and degradation of the environment resulting from their operation. This is particularly important when a system using renewable energy, or a hybrid system, is compared with a conventional system using fossil fuels. Multi-objective optimization methods often involve the definition of arbitrary elements, such as pollution factors, as limits to harmfulness, as well as economic costs.

\subsection{The Common Economic Analysis}

The application of solar thermal systems is often limited to an upper value of the solar fraction, typically $60 \%$. This means that half or more of the energy required for the service, mainly during cold and mid-seasons, is produced using fossil fuels. Even if recognizing the beneficial effects of increasing the value of the solar fraction is straightforward, it is often difficult to demonstrate its economic convenience due to the relatively low cost of the fossil fuel. An increase of the solar fraction corresponds to an increase of the size of the components, the surface of the FPC, the volume of the storage system, and, consequently, the relatively high cost of the SWHS.

The internalization of possible benefits, such as the reduction of greenhouse gas emissions and consequent environmental effects, could make increasing the size of the SWHS a more attractive and viable option. Implementation of particular economic support measures could enhance their convenience, but these are not always sufficient [26].

The analysis of the cost of solar energy systems is not a particularly difficult task. Considering the structure of the system, the total cost is principally dependent on the costs of the five main elements: Solar collectors, storage system, piping, auxiliary heaters, and heat exchangers.

All of these costs can be referred to in a typical structure of the type. The cost of the installation $\left(C_{i n s}\right)$ can be obtained according to [28], considering the various components and the different sizes, by defining cost using the following relation:

$$
C *_{i}=C_{r e f}\left(\frac{X^{*}}{X_{r e f}}\right)^{\alpha}
$$

In this equation, $X_{r e f}$ is the reference size of the component under analysis, $C_{r e f}$ is its reference cost, $X^{*}$ is the scaled value of the component size (higher or lower), and $C_{i}$ is the actual cost of the component. In the case of SWHS, the idea can be applied considering the various elements (collector, boiler, storage tank, piping, or heat exchangers), but the same idea can be applied to the whole system, referring, for example, to a given cost value expressed in Euro for each $\mathrm{kW}$ of power installed. The scaling exponent assumes a value less than unity (the value of many components is in the range between 0.6 and 0.8 ).

Considering a typical SWHS system, for example, it can be reasonable to assume the following reference costs, $C_{\text {ref, }}$, and exponents: $C_{\text {ref }}=800 \mathrm{Euro} / \mathrm{m}^{2}$ and $=0.8$ for the solar collectors; $C_{\text {ref }}=$ $1200 \mathrm{Euro} / \mathrm{m}^{3}$ and $=0.6$ for the storage volume; $C_{r e f}=2500 \mathrm{Euro} / 20 \mathrm{~kW}$ and $=0.75$ for the backup system, using natural gas as fuel. An economic analysis of the system can be obtained, defining three main items: Installation costs of the SWHS, $C_{S W H S}$, connected with the cost of the various components of the system; operating and maintenance costs, $C_{O \& M}$; and cost of the energy source, $C_{\text {res }}$, including both renewable and non-renewable energy sources required for the operation of the system. In the case of solar energy input, it is obviously reasonable to assume this last value as null. The fourth term on the right side of Equation (8) is the operating cost of the auxiliary system, which, in general, is based on fossil fuel or, occasionally, electricity. The function for total cost, defined as the sum of all costs, is given by:

$$
C_{\text {tot }}=C_{S W H S}+C_{O \& M}+C_{r e s}+C_{f u e l} \cdot E_{Q, a u x}
$$


where:

$$
C_{S W H S}=C_{F P C}+C_{V}+C_{\text {boiler }}
$$

The cost of energy produced with the solar system depends on the solar irradiation, on the operating hours of the plant and the amount of energy produced. Considering some market data available, the costs of systems for solar thermal process heat generation in Europe range from 150 to $500 \mathrm{Euro} / \mathrm{m}^{2}$ of collector area. The variation depends on the system architecture and size, typology of the selected components (for example on the typology of collectors used), and other country-specific factors. For medium to large size thermal systems, the costs can be considered as follows:

- $\quad 50 \%$ of the total cost refers to the cost of the collector field, structure, and installation;

- $\quad 20 \%$ of the total cost is connected with piping for the collector field and other parts;

- $\quad 10 \%$ of the cost is connected with the storage system and heat exchanger;

- $\quad 5 \%$ of the cost refers to the auxiliary boiler;

- $\quad 5 \%$ of the cost is connected to the control system;

- $\quad 10 \%$ of the total cost is connected to planning and design activity.

The cost of the system, as defined by Equation (8), assumes significant relevance if considered in conjunction with system productivity. In particular, it can refer to a specific temporal basis (one year or the economic lifetime of the system) so that it can be compared with the energetic derived costs. It is clear that, in the case of fossil fuel-based thermal energy production, the costs of installation are quite low (relating only to the boiler), but the third term, i.e., the cost of resources used, can be significant (for example, in the case of natural gas). On the contrary, in the case of a system based on solar energy input only, the cost of the installation can be relatively high (including cost of collectors, storage systems, auxiliary boiler, and piping system), while the cost of the fuel is zero. An economic compromise between solar energy and the total amount required (mainly the surface of the collectors) often determines a reduced value of the operating solar fraction of the plant (less than $40 \%$ ) and can rule out the choice of installing a solar thermal plant, particularly when subsidies are not available.

\subsection{The Alternative Method for Combined Energetic and Economic Optimum Design of SWHS}

The method proposed here for the optimum design of a solar energy system aimed at increasing the share of solar energy is based on a typical economic analysis, with the introduction of additional costs related to the irreversibility of the fossil fuel backup system and to environmental pollution as a form of additional (penalty) cost.

The operation of an energy system is represented by the scheme of Figure 4, in which energy input and output and the components are not only considered, but also waste heat and exergy losses (energy degradation or irreversibility), as well as pollutant emissions (environmental impact). The main idea of this approach is that, in an analysis of a SWHS, the irreversibility relating to the operation of the auxiliary boilers must assume an economic value. Strictly speaking, for a well-defined load (temperature and temporary profile), the size of the system is defined as a result of the minimization of a cost function; thus, as a result of a multi-objective optimization, considering both economic and energetic variables and the Second Law of Thermodynamics. 


\section{SURROUNDINGS}

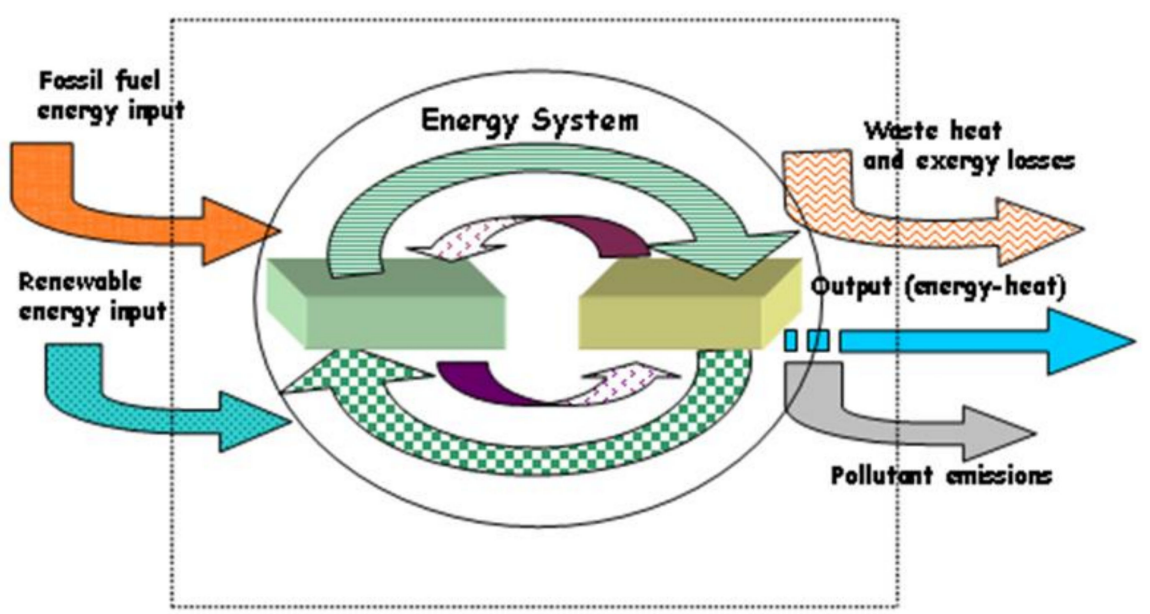

Figure 4. Conceptual point of view for the analysis of an energy system using renewable energies.

For the application of the methodology, the definition of the costs of the solar system is necessary. Three main elements can be identified: The cost of the installation, $\mathrm{C}_{\mathrm{in}}$, including all of the components (collectors, storage volume, fossil fuel backup system, piping); the other operating and maintenance costs, $\mathrm{C}_{\mathrm{O} \& \mathrm{M}}$; and, finally, the cost of the resources used for the operation of the system, including fossil fuel or electricity required for the operation of backup systems, $C_{\text {res. }}$. The total cost can apply to the whole lifetime of the SWHS or on a well-defined temporal basis (for example, one year). The additional idea is to consider the solar fraction $(s f)$ as an independent variable of the problem and express the terms of each objective function in relation to the selected variable.

With respect to the terms already presented in Equation (8), in the new function, the cost related to the irreversibility of the system, $C_{I}$, is added. In addition, all costs depend on the solar fraction, so that, for a given value, the temperature required is the only real independent variable. In this way the total operating cost of the system is:

$$
C_{t o t}(s f)=C_{S W H S}(s f)+C_{O \& M}(s f)+C_{I}(s f)+C_{r e s}(s f)
$$

According to this idea and using the idea expressed in Equation (7), the cost of the components can be expressed as a function of the solar fraction, $s f$ :

$$
C_{F P C}=C_{F P C, r e f}\left(\frac{A_{F P C}(s f)}{A_{F P C, r e f}}\right)^{\alpha_{F P C}} C_{V}=C_{V, r e f}\left(\frac{V_{V}(s f)}{V_{V, r e f}}\right)^{\alpha_{V}}
$$

The power level of the auxiliary heating system is selected that can meet $100 \%$ of the energy demand, to avoid a situation in which a possible shutdown of the solar thermal system corresponds to an interruption in production. For this reason, the cost of the auxiliary boiler is independent of the variable $s f$, and its cost is:

$$
C_{a u x}=C_{a u x, r e f}\left(\frac{P_{a u x}}{P_{a u x, r e f}}\right)^{\alpha_{a u x}}
$$

A comparison of different systems, each characterized by a well-defined collector surface, storage system volume, and fossil fuel auxiliary boiler power, can be undertaken for different values of the solar fraction, $s f$, by analyzing the total operating cost of the system. The minimization of the utility function obtained with Equation (10) allows the definition of an "economic value" of the solar fraction, $s f$, which is the result of the optimum design and not an imposed value. It is expected that, for each case and each operating condition, including the different solar irradiation values, a different share of 
operation of the solar system (expressed by the solar fraction, $s f$ ) with respect to the fossil fuel backup can be obtained.

It is obvious that the typical economic analysis, represented in Figure 5 and expressed by Equation (8), determines a relatively low area of the solar collectors and a corresponding use of the auxiliary boiler. This is due to the relatively low cost of the fuel and the high First Law efficiency (over $90 \%$ ) of the auxiliary boiler. Thus, genuine convenience of the installation of the solar system is possible if and only if economic subsidies are available.

However, the solution based on the simple economic analysis, considering that the same boiler has a very low Second Law efficiency (about 0.1 or less), results in a relatively high value of irreversibility, $\mathrm{I}$, and corresponding carbon dioxide emissions. This is not good from an energy perspective because it underestimates the beneficial effect of solar energy. For this reason, the attribution of an additional cost to the irreversibility (exergy losses) related to the operation of the fossil fuel-based systems, and null in the case of the completely solar-assisted system, allows a compromise between the two opposing objectives (maximizing the solar fraction or minimizing the total cost of the system) to be considered. The method proposed is schematically illustrated in Figure 6 in comparison with the typical economic approach, represented in Figure 5. The idea is that the introduction of a "penalty" cost attached to the irreversibility represents an additional cost, thereby contributing to a further reduction of fossil fuel use and an increase of the solar production share or solar fraction. This cause an increase of the size of the solar plant and a reduction of the operation of the auxiliary boiler, which results in the increase of the value of the solar fraction.

Considering Equation (9), the crucial element for the application of the method appears to be the definition of an appropriate value for the specific cost of irreversibility (exergy losses), $c_{I}$.

Considering a system with only thermal energy output, a cost of irreversibility, defined as a fraction of the fuel price, appears to be the most appropriate choice. The method proposed can be considered a kind of combined "economic and energetic" approach. A similar method has been already applied and discussed by the author of the present paper, for the analysis of Combined Heat and Power (CHP) systems. The objective in the CHP system case is the maximization of its operating share with respect to the auxiliary boiler (fossil fuel backup) [29], and can thus be considered to be similar to the problem addressed in the current paper.

As discussed in the previous sections, referring to a conventional economic analysis, the analysis of an energy system considers only the cost of the input energy ("fuel") and the cost of the various components of the system (including maintenance costs).

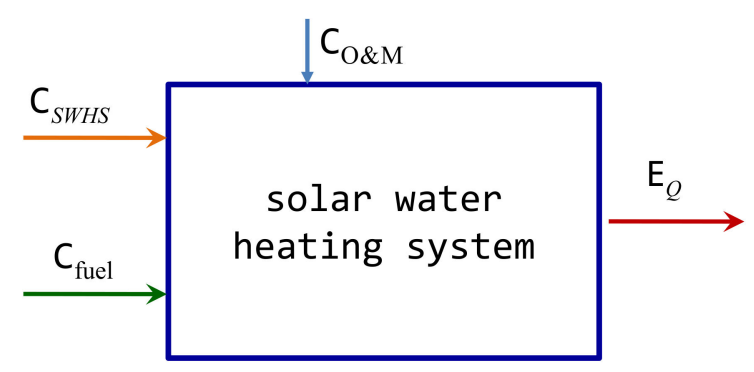

Figure 5. Analysis of a SWHS for process heat with a typical economic approach. 


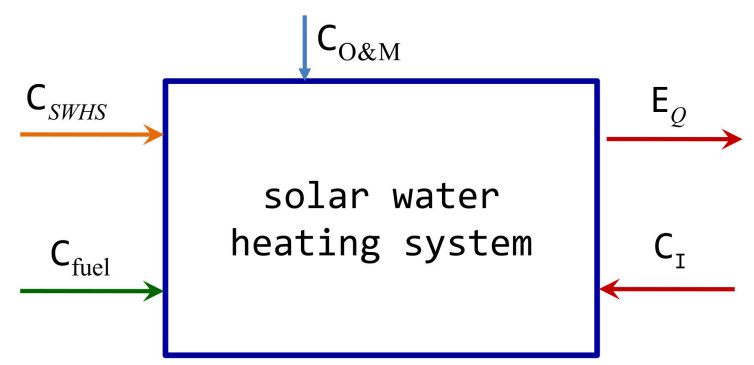

Figure 6. Analysis of a SWHS for process heat with the method proposed.

Thus, due to the relatively high specific cost of solar equipment and the relatively low cost of fossil fuel input, it is often difficult to demonstrate a real economic convenience of solar-based systems for production of industrial process heat in comparison with a system based only on the utilization of fossil fuel. Thus, the promotion of widespread use of renewable energy systems is often due to the availability of subsidies and economic support systems.

From a more general perspective, the system should be analyzed by combining energetic (reducing the use of fossil fuels), environmental (reducing emissions of pollutants, mainly $\mathrm{CO}_{2}$ ), and economic convenience. This is the real motivation of the approach proposed in the present paper. In particular, the idea is to consider not only the conventional costs, but also the cost associated with exergy losses connected to the cost of the operation of the auxiliary boiler.

Considering Figure 6, the total cost for the operation of the SWHS can be determined as the sum of the costs related to plant installation, operation, and maintenance, plus the cost of the energy input (dependent on fuel or electricity used) as in the previous function, and with the introduction of an additional term. This last term takes into account both thermodynamic and environmental elements, represented by the cost of exergy losses associated with the operation of the fossil fuel backup system, I. According to the schematization given in the previous section, the total cost can be expressed as:

$$
C_{t o t}=C_{i n s}+C_{O \& M}+C_{I}+C_{f u e l}=C_{i n s}+C_{O \& M}+c_{I} \cdot I+c_{f u e l} \cdot E_{Q, a u x}+c_{e l} \cdot E_{Q, e l}
$$

The idea is quite simple: With respect to the various costs considered in a typical economic analysis, an additional penalty is attributed to the exergy losses, I, caused by the use of fossil fuel or electricity for the operation of the auxiliary boiler. In this way, the operation of the auxiliary boiler can be reduced, and the share of solar energy increased.

The choice of using irreversibility as a penalty term is now explained. It is well known that if fossil fuel is used to produce hot water beginning at a low temperature, a corresponding amount of energy is degraded (irreversibility). This irreversibility is dependent on the amount of energy used, $E_{Q, a u x}$, and on the irreversibility factor, $\mathrm{i}$, expressed in dimensionless terms and as a function of the temperature of the hot water. The irreversibility, in dimensionless terms, is:

$$
i=\frac{T_{o}}{T_{\text {out }}-T_{o}} \ln \left(\frac{T_{\text {out }}}{T_{0}}\right)
$$

so that:

$$
I=E_{Q, a u x} \cdot i
$$

The model of the SWHS, with the main parameters, is summarized in Figure 7. 


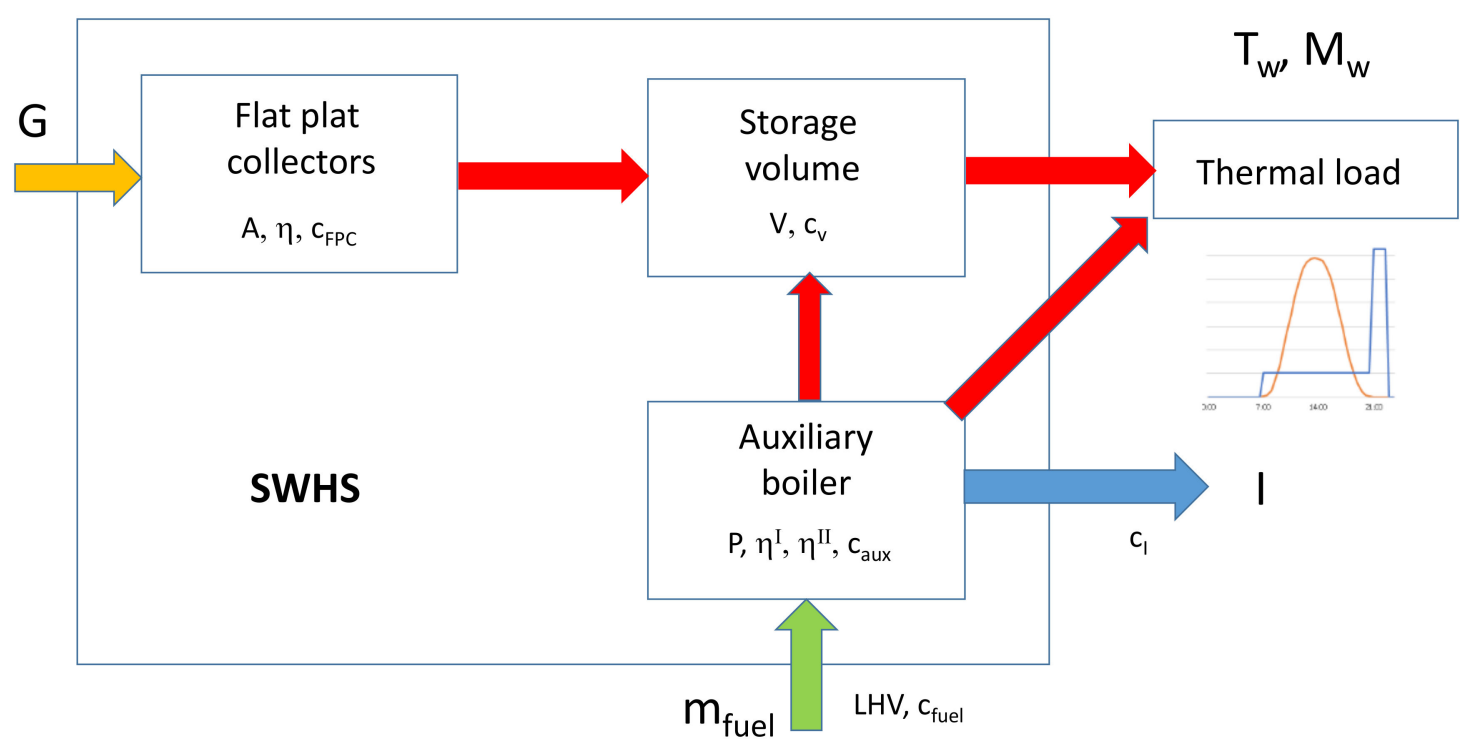

Figure 7. Conceptual flow chart of the method for definition of the optimal size of the SWHS.

Considering Equation (14), $T_{\text {out }}$ is the absolute temperature of the water required (expressed in Kelvin) and $T_{0}$ is a reference absolute temperature (it can also be the inlet temperature of the water). Considering, for example, the case in which $T_{\text {out }}$ is $60{ }^{\circ} \mathrm{C}(333 \mathrm{~K})$ and $T_{0}$ is $15^{\circ} \mathrm{C}(288 \mathrm{~K})$, the irreversibility factor, $\mathrm{I}$, assumes the value of 0.929 . If the temperature increases to the limit value of $100{ }^{\circ} \mathrm{C}$, the irreversibility factor is lower but nonetheless high, equal to 0.875 , meaning that $87.5 \%$ of the energy used is "degraded energy".

Considering the above calculation, it is clear that the introduction of the penalty term, expressed as $c_{I} I$ in Equation (13), is relevant. The key element for defining the real weight of such a term and, consequently, the difference from the results of conventional economic analysis, is the definition of the cost of the exergy losses, $c_{I}$. The same cost can be expressed in dimensionless form as $k_{I}$, using as a reference the cost of one of the input fuels:

$$
k_{I}=\frac{c_{I}}{c_{f u e l}}
$$

The cost of the irreversibility and, consequently, the dimensionless cost expressed by Equation (16), can be defined in different ways, as summarized in Table 2. Considering the four possible options of Table 2, those corresponding to lines 3 and 4 define a kind of "ecological vision" (with a high penalization for the operation of the auxiliary boiler). The expected result is to minimize as much as possible the use of the support backup system with an increase of the surfaces of the solar collectors. On the contrary, the option of line 2 represents a compromise, which partially contributes to a reduction in the use of fossil fuels, while the first option corresponds to the typical economic analysis represented in Figure 5. 
Table 2. Typical values for the cost of irreversibility.

\begin{tabular}{cc}
\hline Penalty Term for Irreversibility & Characteristics \\
\hline$c_{I}=0:$ & $\begin{array}{r}\text { This case corresponds to the conventional economic analysis; no } \\
\text { penalty is considered for the operation of fossil fuel backup systems or } \\
\text { for the associated irreversibility (exergy losses) }\end{array}$ \\
$c_{I}=c_{f u e l} \bar{\eta}_{o}:$ & $\begin{array}{r}\text { The cost of the exergy losses is considered equal to the price of fuel } \\
\text { reduced with a coefficient } \bar{\eta}_{o^{\prime}} \text { representing an average efficiency of the } \\
\text { solar energy systems (e.g., solar collector) }\end{array}$ \\
\hline$c_{I}=c_{f u e l}:$ & $\begin{array}{r}\text { The cost is assumed equal to the cost of the input energy. The cost can } \\
\text { be directly referred to the fuel price, in related units (e.g., Euro/kWh) }\end{array}$ \\
\hline$c_{I}=p_{e l}:$ & $\begin{array}{r}\text { In the case of an auxiliary boiler using electricity, the cost is equal to } \\
\text { the price of electricity }\end{array}$ \\
\hline
\end{tabular}

\section{Application of the Proposed Design Strategy in a Specific Case Study}

The idea of the exposed method is to evaluate, as a monetary cost, all the effects coming from the use of renewable energy, such as solar, and fossil fuels.

To understand the perspectives of the optimum design strategy defined and the different results that can be obtained with the methodology proposed, a specific case is analyzed as a function of the different costs assumed by irreversibility and with respect to the typical economic approaches.

Figure 8 provides the thermal load of a company that requires an amount of hot water for cleaning purposes for each working day of a week. The temperature required for hot water is $65^{\circ} \mathrm{C}$, so that $\mathrm{T}=50^{\circ} \mathrm{C}$. A total daily amount of $10 \mathrm{~m}^{3}$ of water production is required.

The input temperature of cold water is $15^{\circ} \mathrm{C}$. The consumption profile of the company is common, as described in [22]. The profile is the same for five days of the week and for 48 weeks of the year. Considering the typical working day, during the hours from 5:00 to 20:00, a minimum constant level of demand of cleaning water, corresponding to $0.4 \mathrm{~m}^{3} / \mathrm{h}$, is required. In the final part of the day, when all of the production equipment is cleaned before closing time, from 20:00 to 22:00, a higher level of demand of cleaning water is observed, corresponding to $2 \mathrm{~m}^{3} / \mathrm{h}$. Total energy of approximately $583 \mathrm{kWh}$ per day and about 140,000 kWh per year is required. A production of $2 \mathrm{~m}^{3} / \mathrm{h}$ is used to define the size of the auxiliary boiler.

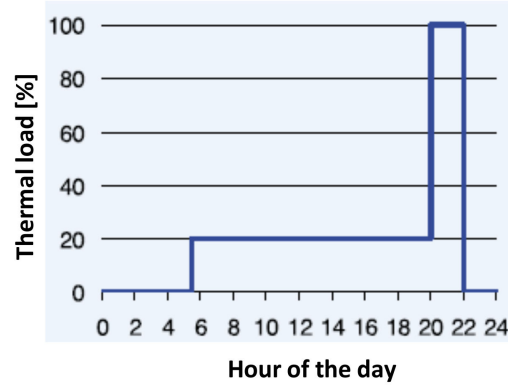

(a)

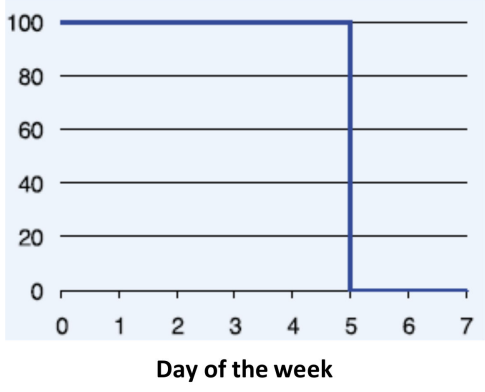

(b)

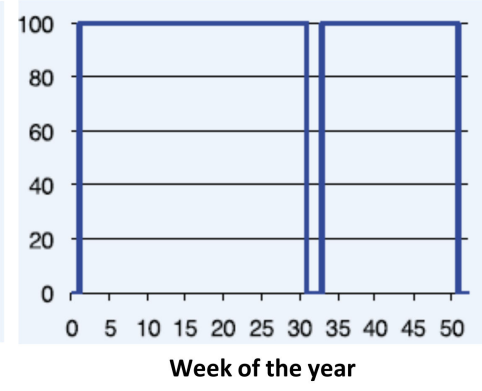

(c)

Figure 8. Example of a typical load profile for hot water demand for cleaning purposes in a factory for a single day (a), for a week (b), and for a year (c).

The analysis is based on the installation of the SWHS in a location in which the annual solar irradiation is about $1500 \mathrm{kWh} / \mathrm{m}^{2}$. The economic life of the plant and of the components is fixed at 20 years, so that it is possible to define an annual operational cost of the system. Tables 3 and 4 contain the main economic data for the calculation, while Table 5 and Figure 9 provide the results obtained in 
the three different cases. Analyzing the results, it is possible to observe that the optimal value of the solar fraction, $s f$, varies from 0.34 (case 1), a typical value suggested in the literature, up to 0.83 (case 3).

For the three cases analyzed, Table 5 provides the corresponding area of the solar collectors and the amount of fossil fuel energy required from fossil fuel backup; this last value is obtained considering a lower heating value of $10 \mathrm{kWh} / \mathrm{m}^{3}$ and an average efficiency of the auxiliary boiler of 0.9 . As is evident from Table 5, the area of the solar collector increases from case $1\left(96 \mathrm{~m}^{2}\right)$, in which no penalty is given to the operation of the auxiliary boiler, to case $3\left(234 \mathrm{~m}^{2}\right)$, in which the maximum penalty for the operation of the same boiler is considered. The volume of the storage system in the three cases under analysis ranges from 10 to $15 \mathrm{~m}^{3}$. The three solutions obviously correspond to three different values of the share of operation with solar energy, namely the solar fraction, $s f$. The solar fraction, $s f$, is strongly influenced by the introduction of a penalty cost of irreversibility. Case 4 of Table 2, concerning the plant equipped with an auxiliary boiler operating with electricity, is not considered, even if it is clear that the optimized value of the solar fraction could be shifted towards values higher than 0.8 .

Figure 9 compares the qualitative trends of the utility function "annual economic cost" of the system, defined with Equation (10) and Equation (13), considering the different values of the cost of the exergy losses. The orange curve represents the "fuel cost" function, the blue curve the "equipment cost", and the "cost of irreversibility" is colored gray (obviously absent in the left graphs, which relate to the simple economic analysis). Analyzing Figure 9 in detail, it is possible to observe how the "annual economic cost" function is composed mainly of two groups of terms, which present opposite trends according to the independent variable, $s f$ : The cost of the solar thermal system (which increases) and the cost of the fuel used by the auxiliary heating system (which decreases). As parameter $\mathrm{k}$ increases, the economic penalty attributed to irreversibility (exergy losses) produced in the combustion process increases. Analyzing Figure 8, it is possible to appreciate how, in the case of a simple economic analysis, when the cost of the irreversibility $c_{I}$ is null, the SWHS does not appear to be convenient when an economic support system is not available. A reduced effect is determined by the use of solar energy and the whole cost of the system remains the same for solar fractions ranging from 0 to 0.34 . For solar fractions higher than 0.34 , the cost of the system increases; thus, in this particular case, the maximum value of the solar fraction can be considered equal to 0.34 . This value can be considered relatively low with respect to that suggested in the main technical standards (0.4). This is due to the relatively high cost of solar systems, however, it is similar to the value suggested in the literature.

Table 3. Typical values for application of Equation (8).

\begin{tabular}{cccc}
\hline Component & Reference Size, $\boldsymbol{X}_{\boldsymbol{r e f}}[\mathrm{X}]$ & Reference Cost, $\boldsymbol{C}_{\boldsymbol{r e f}}[\boldsymbol{\epsilon}]$ & $\begin{array}{c}\text { Scaling Exponent for } \\
\text { the Cost Function, }[-]\end{array}$ \\
\hline FPC and piping system & $1 \mathrm{~m}^{2}$ & 800 & 0.8 \\
Storage volume & $1 \mathrm{~m}^{3}$ & 1200 & 0.6 \\
Auxiliary boiler & $20 \mathrm{~kW}$ & 2500 & 0.75 \\
\hline
\end{tabular}

Table 4. Typical values for application of Equation (13).

\begin{tabular}{ccc}
\hline & Reference Cost [-] & Real Cost [€/kWh] \\
\hline Cost of natural gas & 1 & 0.04 \\
Cost of Irreversibility (Economic vision) & $\mathrm{k}=0$ & 0 \\
Cost of Irreversibility (Compromise vision) & $\mathrm{k}=0.35$ & 0.014 \\
Cost of Irreversibility (Ecological vision) & $\mathrm{k}=1$ & 0.04 \\
\hline
\end{tabular}


Table 5. Area of the solar collectors and natural gas consumption in the three cases under analysis described in Table 4.

\begin{tabular}{cccccc}
\hline & $\mathbf{k}_{\mathbf{I}}$ & $\begin{array}{c}\text { Optimal Solar } \\
\text { Fraction (sf) }\end{array}$ & $\mathbf{A}\left[\mathbf{m}^{\mathbf{2}}\right]$ & $\mathbf{V}\left[\mathbf{m}^{3}\right]$ & $\begin{array}{c}\text { Natural Gas } \\
\left.\text { Consumption [m }{ }^{3}\right]\end{array}$ \\
\hline Economic vision & 0 & 0.34 & 96 & 10 & 10,260 \\
\hline Compromise vision & 0.35 & 0.58 & 162 & 13 & 4975 \\
\hline Ecological vision & 1 & 0.82 & 234 & 15 & 2643 \\
\hline
\end{tabular}

$\mathrm{C}_{\text {tot }}$

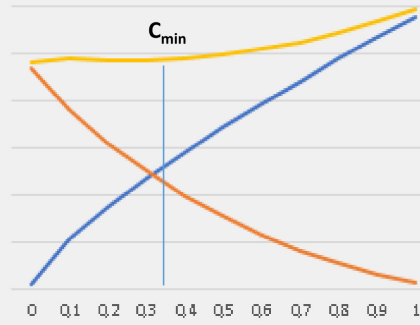

sf

(a)

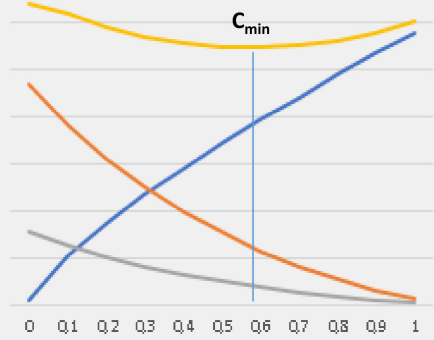

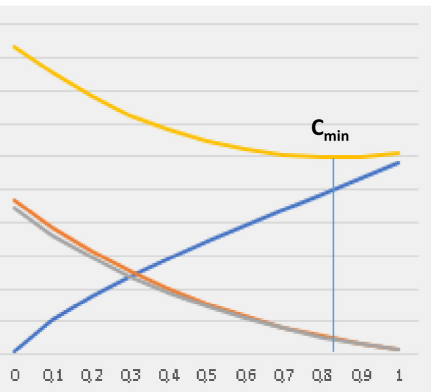

sf

(b)

(c)

Figure 9. Optimal value of solar fraction obtained with different costs of the irreversibility corresponding to economic perspective (a), compromise (b), and ecological vision (c).

When a penalty is introduced for the energy degradation (exergy losses), if $c_{I}$ is higher than zero (cases $b$ and $c$ ), two effects can be remarked upon. The function $C_{\text {TOT }}$ has a minimum, obtained for a defined value of the solar fraction; moreover, the absolute minimum of the utility function shifts towards increasing values of the solar fraction, sf, meaning a higher surface area of solar collectors, as the penalty term increases. Thus, the idea of penalization of the irreversibility connected to the operation of the auxiliary boiler encourages the development of a SWHS with increasing values of the solar fraction, $s f$. In this way, the result is an increase of the surface of the collectors and a corresponding reduction of fossil fuel use. It is clear that the method proposed, although penalizing the use of fossil fuels, does not exclude it a priori; on the contrary, their importance is recognized in order to support the system in critical situations or when a reduced amount of energy is available.

\section{Discussion}

In approaching this study, the author considers the problem of promoting and increasing the use of solar energy in the industrial sector. The attempt made in the paper is to develop a simple method for supporting a further promotion of solar energy in the industrial sector. This is mainly for the production of thermal energy in the form of hot water at temperatures below $100{ }^{\circ} \mathrm{C}$, an application that is of relevant importance, and for which the use of FPCs is proposed.

Industries clearly also have higher temperature needs. The production of process heat with solar collectors for temperature levels between 100 and $300{ }^{\circ} \mathrm{C}$ was studied, combining parabolic trough solar collectors coupled to a storage tank and auxiliary heat source in a recent paper [30].

Previous studies agree with the idea that solar energy, which could fulfil low-temperature industrial heat demands with relevant potential in high solar irradiance countries, is an underutilized resource, as well represented by reviews [2-4]. In addition, further increase is expected in the coming years.

Medium to low temperature production (water at temperatures in the range between 40 and $100{ }^{\circ} \mathrm{C}$ ), in particular, can be strongly recommended for the use of solar energy. 
Recent studies have proposed new systematic methods for the optimization of solar heat integration with different process options. However, other recent papers have aimed at minimizing the levelized cost of heat produced, combining the different methods available [31].

The proposed method is not an alternative to the existing methods available in the literature based on economic analysis; rather, it is an integrative approach. In particular, it tries to present a different perspective that may be useful for analyzing the performance of hybrid systems, based on renewable and fossil sources. Although it must, therefore, be considered integrative, the presented approach has the same objective: To support the promotion of solar energy for process heat and provide possible assistance in the definition of alternative instruments for energy policy, according to the requirement of countries that are lacking a primary energy source.

From this perspective, the method proposed appears to be an easily applicable instrument to support the design of SWHSs for process heat production. The case discussed and the example presented consider a typical situation in which water at a relatively low temperature $\left(65^{\circ} \mathrm{C}\right)$ is required.

All systems that use solar energy require a fossil fuel backup. Moreover, it is necessary to permit operation under various conditions, and the size of the various components of a solar system are often relatively small. Thus, the author has shown that the solar fraction can be obtained in the form of an optimization variable.

From the analysis, it is evident that the penalization imposed on the operation of the fossil fuel backup system, expressed in terms of a monetary cost, $c_{I}$, or in dimensionless terms, $k_{I}$, is relevant in defining the size of the solar system and, in particular, the FPC surface. For example, considering the specific case analyzed in Section 5, the optimized solution, in terms of collector surface, could vary by a factor of 2.4 (from $96 \mathrm{~m}^{2}$ in the case of economic analysis to $234 \mathrm{~m}^{2}$ ) if the maximum penalty is attributed to the irreversibility associated with the operation of the auxiliary boiler. The results of the analysis discussed in Section 5 are relative to well-defined values of the cost of the components. The author intentionally considered relatively high costs for the solar system; nonetheless, apart from the specific choice, the results obtained in terms of the shift of the optimum value of the operating share of the solar energy use with respect to fossil fuel is relevant.

\section{Conclusions}

Further development and additional use of solar thermal energy, mainly for low to medium temperature industrial process heat production, particularly that below $100{ }^{\circ} \mathrm{C}$, can be linked to a compromise between positive elements, such as the reduced cost of energy input and minor pollutant emissions, and negative elements, such as the high installation costs of the components of the SWHS.

According to the literature, the trade-off is possible with hybrid systems (combining the use of fossil fuels and renewable energy) with a well-defined value of the "solar fraction", referring to a whole year. This value ranges from 0.4 and 0.6 , and only in some particular cases can overcome the upper limit. The exact value depends on the technical elements (temperature required, characteristics of the load), environmental elements (solar irradiation, environmental temperature), and the economic cost of the system.

The idea proposed in the paper is to combine, on a common basis (i.e., an operational cost), economic, energetic, and environmental elements. The objective is to pursue an appropriate balance between the inefficiencies of the system, represented by the exergy losses associated with the use of fossil energy (for the auxiliary boiler), and economic costs. This is obtained through the introduction of an additional term, the cost of the irreversibility, that represents a penalty on the use of the fossil fuel backup system necessary to support the system in case of reduced solar energy input, such as during winter seasons or days with reduced low solar irradiation. In this way, it is possible to determine an exact quantification of the environmental impact of the system.

The application of the method in a typical case, characterized by medium temperature $\left(65^{\circ} \mathrm{C}\right)$ and relatively high solar irradiation $\left(1500 \mathrm{kWh} / \mathrm{m}^{2}\right)$, demonstrates the importance of the cost applied to the irreversibility. By modifying this cost, a corresponding increase of the optimum solar fraction of the SWHS is observed, moving from a value of 0.3-0.4 (in the case of economic analysis) to values as 
high as 0.8 when the penalty cost attributed to the irreversibility is equivalent to the cost of the fuel. It is important to emphasize that the maximum level of the solar fraction is never higher than $90 \%$, which appears to be correct; although the use of fossil fuels is penalized, it is not convenient to exclude a priori their contribution.

The optimal value of the solar fraction depends obviously on the temperature required for the process heat, the solar variables (mainly the solar irradiation and efficiency of solar collectors), the cost structure of the various components, and particularly the definition of the cost of irreversibility. The optimal value of the solar fraction, $s f$, is the result of the analysis and will be different in various cases. The application of the proposed methodology could be important to promote possible further economic support policies, from the perspective of obtaining a corresponding increase in the use of solar energy for process heat, mainly in medium to low temperature applications $\left(40-100^{\circ} \mathrm{C}\right)$, for which FPC can be used.

Funding: The author would like to express appreciation to the University of Pisa for the research grant "Fondi di Ateneo". This research received no external funding except those coming from the University of Pisa.

Acknowledgments: Author would like to acknowledge Giacomo Bertola, a former student of Energy Engineering at the University of Pisa, for his contribution to the presented activity, that was partially object of his dissertation Thesis, discussed during year 2019.

Conflicts of Interest: The author declares no conflict of interest.

\section{Nomenclature}

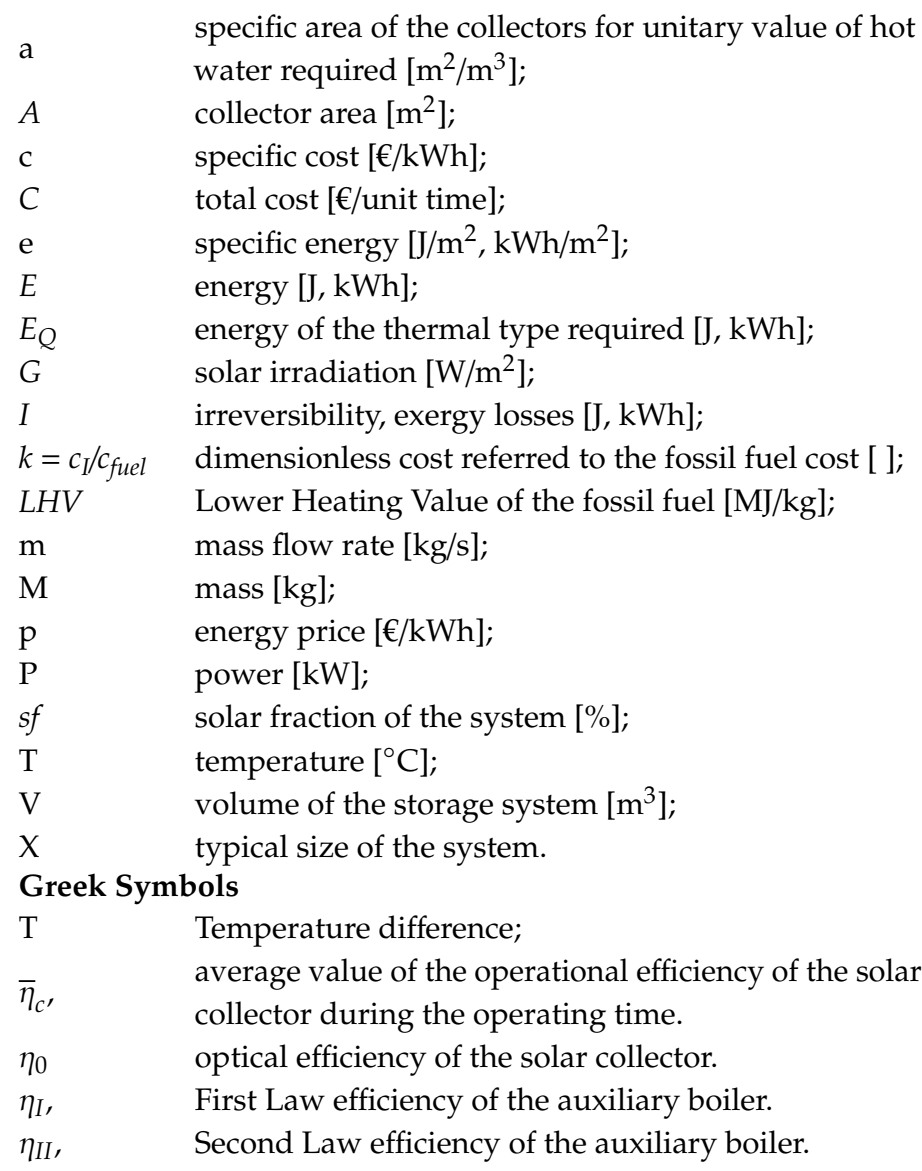




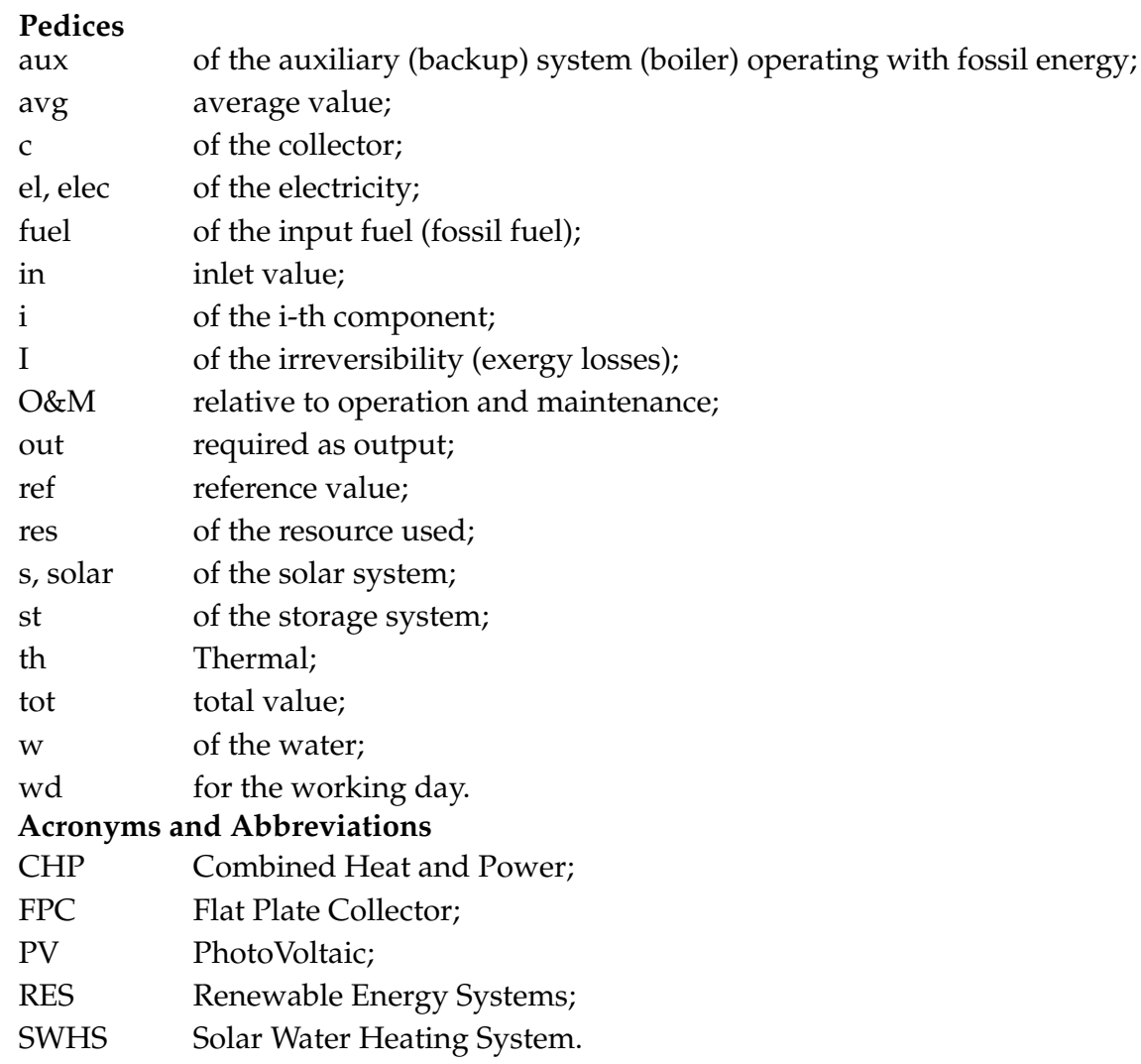

\section{References}

1. Kalogirou, S. The potential of solar industrial process heat applications. Appl. Energy 2003, 76, 337-361. [CrossRef]

2. Sharma, A.K.; Sharma, C.; Mullicka, S.C.; Kandpal, T.C. Solar industrial process heating: A review. Renew. Sustain. Energy Rev. 2017, 78, 124-137. [CrossRef]

3. Farjana, S.H.; Huda, N.; Parvez Mahmud, M.A.; Saidur, R. Solar process heat in industrial systems-A global review. Renew. Sustain. Energy Rev. 2018, 82, 2270-2286. [CrossRef]

4. Kumara, L.; Hasanuzzamana, M.; Rahima, N.A. Global advancement of solar thermal energy technologies for industrial process heat and its future prospects: A review. Energy Convers. Manag. 2019, 195, 885-908. [CrossRef]

5. Duffie, J.A.; Beckman, W.A. Solar Engineering of Thermal Processes; John Wiley: New York, NY, USA, 1980.

6. Kreith, F.; Yogi Goswami, D. Handbook of Energy Efficiency and Renewable Energy; CRC Press Taylor \& Francis: New York, NY, USA, 2016; pp. 916-970.

7. Sturm, B.; Meyers, S.; Zhang, Y.; Law, R.; Siqueiros Valencia, E.J.; Bao, H.; Wang, Y.; Chen, H. Process intensification and integration of solar heat generation in the Chinese condiment sector-A case study of a medium sized Beijing based factory. Energy Convers. Manag. 2015, 106, 1295-1308. [CrossRef]

8. Klein, S.A.; Beckman, A. A general design method for Closed-Loop Solar Energy System. Sol. Energy 1979, 22, 269-282. [CrossRef]

9. Braun, J.E.; Klein, S.A.; Pearson, K.A. An improved design method for Solar Water Heating System. Sol. Energy 1983, 31, 597-604. [CrossRef]

10. Collares-Pereira, M.; Gordon, J.M.; Rabl, A.; Zarmi, Y. Design and optimization of solar industrial hot water system with storage. Sol. Energy 1984, 32, 121-133. [CrossRef]

11. Gordon, J.M.; Zarmi, Y. An analytic model for the long-term performance of solar thermal system with well-mixed storage. Sol. Energy 1985, 35, 55-61. [CrossRef]

12. Eskin, N. Performance analysis of a solar process heat system. Energy Convers. Manag. 2000, 41, 1141-1154. [CrossRef] 
13. Tilahun, F.B.; Bhandari, R.; Mamo, M. Design optimization and control approach for a solar-augmented industrial heating. Energy 2019, 179, 186-198. [CrossRef]

14. Reddy, T.A.; Gordon, J.M.; De Silva, I.P.D. Mira: A one-repetitive day method for predicting the long-term performance of solar energy system. Sol. Energy 1987, 39, 123-133. [CrossRef]

15. Kulkarni, G.N.; Shireesh, B.K.; Santanu, B. Determination of design space and optimization of solar water heating systems. Sol. Energy 2007, 81, 958-968. [CrossRef]

16. Renaldi, R.; Friedrich, D. Techno-economic analysis of a solar district heating system with seasonal thermal storage in the UK. Appl. Energy 2019, 236, 388-400. [CrossRef]

17. Antoniadis, C.N.; Martinopoulos, G. Optimization of a building integrated solar thermal system with seasonal storage using TRNSYS. Renew. Energy 2019, 137, 56-66. [CrossRef]

18. Kubinski, K.; Szabłowski, Ł. Dynamic model of solar heating plant with seasonal thermal energy storage. Renew. Energy 2020, 145, 2025-2033. [CrossRef]

19. Huang, J.; Fan, J.; Furbo, S.; Chen, D.; Dai, Y.; Kong, W. Economic analysis and optimization of household solar heating technologies and systems. Sustain. Energy Technol. Assess. 2019, 36, 100532. [CrossRef]

20. Mousa, O.B.; Taylor, R.A.; Shirazi, A. Multi-objective optimization of solar photovoltaic and solar thermal collectors for industrial rooftop applications. Energy Convers. Manag. 2019, 195, 392-408. [CrossRef]

21. Karki, S.; Haapala, K.R.; Fronk, B.M. Technical and economic feasibility of solar flat-plate collector thermal energy systems for small and medium manufacturers. Appl. Energy 2019, 254, 113649. [CrossRef]

22. Viessmann, M. 2008 Solar Thermal Systems-Technical Guide, Viessmann Werke. Available online: www.viessmann.com (accessed on 8 June 2020).

23. Solar Process Heat Generation: Guide to Solar Thermal System Design for Selected Industrial Processes, SO-PRO: Solar Process Heat. Available online: www.solar-process-heat.eu (accessed on 8 June 2020).

24. Lauterbach, C.; Schmitt, B.; Vajen, K. System analysis of a low-temperature solar process heat system. Sol. Energy 2014, 101, 117-130. [CrossRef]

25. Abikoye, B.; Cucek, L.; Isafiade, A.J.; Kravanja, Z. Integrated design for direct and indirect solar thermal utilization in low temperature industrial operations. Energy 2019, 182, 381-396. [CrossRef]

26. Kalogirou, S.A. Solar Energy Engineering: Processes and Systems; Elsevier: Amsterdam, The Netherlands, 2013.

27. Peters, M.; Schmidt, T.; Wiederkehr, D.; Schneider, M. Shedding light on solar technologies-A techno-economic assessment and its policy implications. Energy Policy 2011, 39, 6422-6439. [CrossRef]

28. Bejan, A.; Tsatsaronis, G.; Moran, M.J. Thermal design and optimization; John Wiley: New York, NY, USA, 1996.

29. Franco, A.; Bellina, F. Methods for optimized design and management of CHP systems for District Heating Networks (DHN). Energy Convers. Manag. 2018, 172, 21-31.

30. Bellos, E.; Daniil, I.; Tzivanidis, C. Energetic and Financial Optimization of Solar Heat Industry Process with Parabolic Trough Collectors. Designs 2018, 2, 24. [CrossRef]

31. Sing, C.K.L.; Lim, J.S.; Walmsley, T.G.; Liew, P.Y.; Goto, M.; Zaki, S.A.; Salim, B.S. Time-Dependent Integration of Solar Thermal Technology in Industrial Processes. Sustainability 2020, 12, 2322. [CrossRef]

(C) 2020 by the author. Licensee MDPI, Basel, Switzerland. This article is an open access article distributed under the terms and conditions of the Creative Commons Attribution (CC BY) license (http://creativecommons.org/licenses/by/4.0/). 